\title{
Accessory tricuspid valve tissue
}

INSERM

\section{Source}

INSERM. (1999). Orphanet: an online rare disease and orphan drug data base. Accessory tricuspid valve tissue. ORPHA:95462

Accessory tricuspid valve tissue is a rare, congenital, atrioventricular valve malformation characterized by fixed or mobile accessory tissue on the tricuspid valve, usually associated with other complex cong enital heart anomalies (atrial septal defect, ventricular septal defect, transposition of great arteries, tetralogy Fallot). It may present clinically with systolic murmur, dyspnea, cyanosis, depending also on accompanying congenital heart anomaly. 